\title{
Lymphoscintigraphy with peritumoral injection versus lymphoscintigraphy with subdermal periareolar injection of technetium-labeled human albumin to identify sentinel lymph nodes in breast cancer patients
}

\author{
Giuseppe Caruso', Calogero Cipolla ${ }^{2}$, Renato Costa ${ }^{3}$, \\ Alessandra Morabito ${ }^{3}$, Stefania Latteri', Salvatore Fricano ${ }^{2}$, \\ Sergio Salerno' and Mario Adelfio Latteri ${ }^{2}$
}

\begin{abstract}
Background: Preoperative lymphoscintigraphy is without doubt a valid method for the detection of the sentinel lymph node (SLN). There has been considerable debate regarding the optimal site for the introduction of the tracer; various sites include peritumoral (PT), periareolar (PA), subdermal, and intradermal injection.

Purpose: To evaluate retrospectively the lymphoscintigraphic identification rate of peritumoral (PT) injection versus subdermal periareolar (PA) injection in the detection of SLNs in breast cancer.

Material and Methods: Between October 2002 and December 20I I, a cohort of 906 consecutive patients with biopsy proven breast cancer underwent 914 SLN biopsy procedures. A total of 339 procedures (Group A) were performed using a PT deep injection of radiotracer while 575 procedures (Group B) adopted a subdermal PA injection of radiotracer towards the upper outer quadrant, regardless of the site of the carcinoma. All the patients underwent synchronous excision of the breast cancer and SLN biopsy.

Results: SLNs were identified in the lymphoscintigram in $308 / 339$ cases $(90.85 \%)$ of Group A (PT injection) and in $537 /$ 575 cases $(93.39 \%$ ) of Group B (PA injection). Furthermore, in 2/339 patients (0.58\%) of Group A, internal mammary lymph nodes were found at lymphoscintigraphy, whereas no internal mammary sentinel nodes were found in the Group B patients. The intraoperative identification rate of axillary SLNs was $99.41 \%$ (337 of 339) in the Group A patients and 99.65\% (573 of 575) in the Group B patients. There was no significant difference in the two groups between the incidence of the number of SLNs detected and the incidence of identification of positive SLNs.

Conclusion: PT versus PA injection of radiotracer showed comparable success rates for axillary SLN identification, and can be considered a rapid and reliable method.
\end{abstract}

\section{Keywords}

Breast cancer, sentinel lymph node biopsy, lymphoscintigraphy, sub areolar, peritumoral, radio-colloid

Date received: 19 October 2012; accepted: 12 May 2013

The sentinel lymph node biopsy (SLNB), was first introduced in the 1990s (1-3) and during recent years has become more and more accepted as a method of accurate axillary lymph node staging for breast cancer (4-9). The sentinel lymph node (SLN) is defined as the first lymph node in a regional basin that receives lymph flow from the primary tumor and it is thus the node
'Department of Medical Biotechnologies and Forensic Medicine - Section of Radiological Sciences, AOU Policlinico Paolo Giaccone - University of Palermo, Palermo, Italy

${ }^{2}$ Department of Oncology, Division of General and Oncological Surgery, AOU Policlinico Paolo Giaccone - University of Palermo, Palermo, Italy ${ }^{3}$ Department of Internal Medicine, Division of Nuclear Medicine, AOU Policlinico Paolo Giaccone - University of Palermo, Palermo, Italy

Corresponding author:

Giuseppe Caruso, University of Palermo, via del Vespro, 127 Palermo, 90144, Italy.

Email: giuseppe.caruso@unipa.it 
with the highest probability of being involved in the case of lymphatic spread from the tumor.

Today SLNB represents a minimally invasive operative procedure that can accurately predict the status of axillary lymph nodes in clinical node-negative breast cancer patients and represents an alternative to conventional axillary lymph node dissection. The lymph drainage of the breast is relatively confined to the axilla but at times, though rarely, isolated lymphatic drainage of internal mammary chain-SLNs is reported (2.6-4\%), and most surgeons therefore not always perform the routine removal of extra-axillary nodes. $(10,11)$.

When the SLNB technique was introduced for the staging and treatment of breast cancer, preoperative lymphoscintigraphy was introduced accordingly (1$3,12,13)$. There has been some debate about the optimal site for the introduction of the tracer. The various sites of injection include peritumoral (PT), periareolar (PA), subdermal, and intradermal injection $(14,15)$. Despite such a wide variety of modalities utilized for tracer injection, the high rate of SLNs identification and the low incidence of false-negative cases are surprisingly homogeneous (16). This observation has led to speculations that the site of injection is relatively unimportant, because the entire breast appears to drain to the same SLN $(17,18)$.

The aim of our study was to evaluate retrospectively the lymphoscintigraphic identification rate of different radio colloid injection techniques, comparing PT injection versus subdermal PA injection in the detection of SLNs in breast cancer.

\section{Material and Methods}

A retrospective study was conducted on a cohort of 906 consecutive patients with biopsy proven breast cancer that underwent 914 SLNB procedures between October 2002 and December 2011. Eight patients had bilateral breast cancer. Patients with ductal carcinoma in situ diagnosed at needle core biopsy and patients with multifocal breast cancers were included in the study. Patients with cytological positive lymph nodes, with previous ipsilateral axillary surgery, and patients who received neoadjuvant chemotherapy before surgery were excluded from the study.

\section{Lymphoscintigraphy}

The lymphoscintigraphy injection protocol differed in the study period. From October 2002 to August 2006, 339 procedures (Group A) were performed using a PT deep injection of radiotracer in the breast parenchyma at four points. For non-palpable tumors the injection was performed using ultrasound or stereotactic guidance. From September 2006 to December 2011, 575 procedures (Group B) were performed using a subdermal PA injection of radiotracer towards the upper outer quadrant, regardless of the site of the carcinoma.

Lymphoscintigraphy was performed in the two groups of patients at least 15,30 , and $180 \mathrm{~min}$ after the administration of $40-60 \mathrm{MBq}{ }^{99 \mathrm{~m}} \mathrm{Tc}-$ labeled colloidal albumin in physiologic saline $\left(\mathrm{Nanocoll}^{\circledR}, \mathrm{GE}\right.$ Healthcare, Chicago, IL, USA). Images of the thoracic region at anterior and oblique projections, including the involved breast and axillary area, were acquired using a digital gamma camera computer system with a low-energy high-resolution collimator and an energy peak at $140 \mathrm{KeV}$ (General Electric Millennium MG Gamma Camera Imaging System, Millennium GE, Milwaukee, WI, USA). Only the first hot spots outside the injection site were identified as SLNs. Subsequently a cobalt pen was used to locate the cutaneous projection of the SLN, and the skin overlying the hot spot was marked with a skin marker.

\section{Surgical procedure}

All the patients underwent synchronous excision of the breast cancer, either by conservative surgery or total mastectomy, and SLN biopsy. In cases of non-palpable breast lesions, a guidewire was placed in the tumor site under either stereotactic or ultrasonographic guidance.

For the intraoperative identification of the SLN a radio-guided surgical probe (Neoprobe neo2000 Gamma Detection Systems ${ }^{\circledR}$ - Neoprobe Corporation, Dublin, OH, USA) was used. In a very few cases, where the radio-guided surgical probe picked up a weak radiotracer signal, about $10 \mathrm{~min}$ before the beginning of surgery a subdermal PA injection of $0.5-0.8 \mathrm{~mL}$ of vital stain (Lymphazurin 1\%) was performed. All hot and/ or blue lymph nodes were removed.

All SLNs were examined at frozen section. SLNs with a diameter $<5 \mathrm{~mm}$ were frozen intact, whereas nodes with a diameter $>5 \mathrm{~mm}$ were bisected longitudinally and frozen. Two $4 \mu \mathrm{m}$ frozen sections were taken from the optimal cross-sectional surface of one half of the lymph node and stained with Hematoxylin and Eosin (H\&E) for intraoperative examination. For definitive histopathological examination, all the remaining tissue was formalin-fixed and paraffinembedded.

All patients with SLN metastasis in frozen section (FS) underwent immediate completion of total axillary lymph nodes dissection (ALND). If the SLN was negative at FS but was found to contain metastasis in the final histopathology, patients underwent delayed completion of total ALND. No completion ALND was performed in women with metastasis-free SLNs. 


\section{Statistical methods}

Chi-square test was performed to compare the rate between the different groups.

$P<0.05$ was considered to be statistically significant.

\section{Results}

The mean age of the patients was of 56.1 years (range, 35-81 years) in group A and of 54.7 years (range, 31-80 years) in group B. The features of the 914 tumors are indicated in Table 1.

The average size of the tumors, evaluated on the widest lesion, was $19.7 \pm 10.4 \mathrm{~mm}$ (range, $4-53 \mathrm{~mm}$ ) in group A and of $21.2 \pm 12.4 \mathrm{~mm}$ (range, $6-57 \mathrm{~mm}$ ) in group B.

The lymphoscintigram demonstrated SLNs in 308 of the 339 cases $(90.85 \%)$ of Group A (PT injection) (Fig. 1) and in 537 of the 575 cases $(93.39 \%)$ of Group B (PA injection) (Fig. 2) (chi-square, 1.96; $P=0.16)$

Furthermore, in two of the 339 patients $(0.58 \%)$ of Group A, internal mammary lymph nodes were found at lymphoscintigraphy, whereas no internal mammary sentinel nodes were found in the Group B patients. No differences in progression time of the radiotracer from the injection site were observed between the two groups of patients.

Table I. Features of the 914 tumors.

\begin{tabular}{lrrrr}
\hline & Group A & \multicolumn{3}{c}{ Group B } \\
\hline Mean age (years) & 56.1 & (range, & 54.7 & (range, \\
& & $35-81)$ & & $31-80)$ \\
Upper outer quadrant & 188 & $55.6 \%$ & 319 & $55.5 \%$ \\
Upper inner quadrant & 64 & $18.9 \%$ & 112 & $19.5 \%$ \\
Lower outer quadrant & 47 & $13.9 \%$ & 78 & $13.5 \%$ \\
Lower inner quadrant & 31 & $9.1 \%$ & 47 & $8.2 \%$ \\
Central & 9 & $2.5 \%$ & 19 & $3.3 \%$ \\
Bilateral breast cancer & 3 & $0.88 \%$ & 5 & $0.87 \%$ \\
PTIa & 20 & $5.9 \%$ & 38 & $6.6 \%$ \\
PTIb & 76 & $22.4 \%$ & 127 & $22.1 \%$ \\
PTIc & 146 & $43.1 \%$ & 246 & $42.8 \%$ \\
PT2 & 92 & $27.1 \%$ & 155 & $26.9 \%$ \\
PT3 & 5 & $1.5 \%$ & 9 & $1.6 \%$ \\
Invasive ductal & 234 & $69.1 \%$ & 403 & $70.1 \%$ \\
Invasive lobular & 27 & $8.0 \%$ & 51 & $8.9 \%$ \\
Invasive ductal & 6 & $1.7 \%$ & 11 & $1.9 \%$ \\
$\quad+$ lobular & & & & \\
Other & 58 & $17.1 \%$ & 92 & $16.0 \%$ \\
Ductal carcinoma in situ & 14 & $4.1 \%$ & 18 & $3.1 \%$ \\
\hline
\end{tabular}

The intraoperative identification rate of axillary SLNs was $99.41 \%$ (337 of the 339 patients) in the Group A patients. In 29 of the patients, pre-operative lymphoscintigraphy did not identify the SLNs, which were, however, identified intraoperatively. In the Group B patients the intraoperative identification rate of axillary SLNs was 99.65 (573 of the 575 patients). In

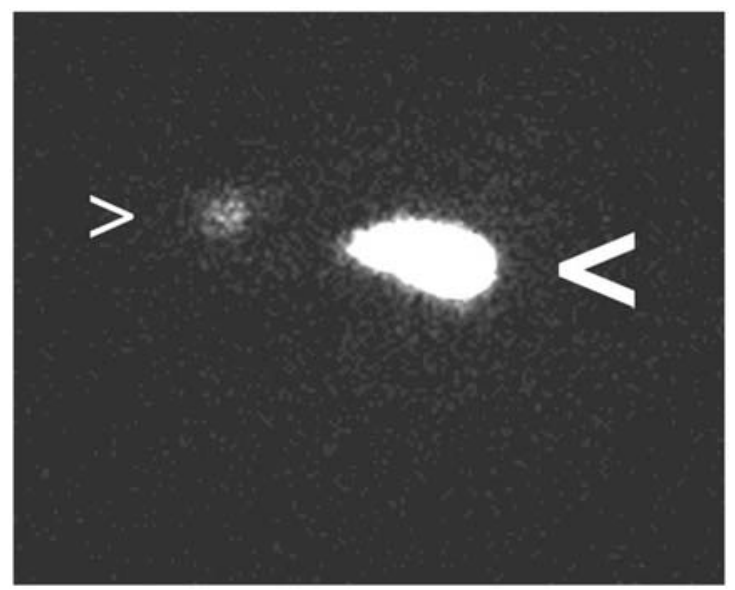

Fig. I. Lymphoscintigraphy of the right breast: the large arrow indicates the injection site of the radiotracer within the tumor site. It can be seen that the radiotracer also tends to spread in the peritumoral area with the typical "drop" morphology. The small arrow indicates the SLN.

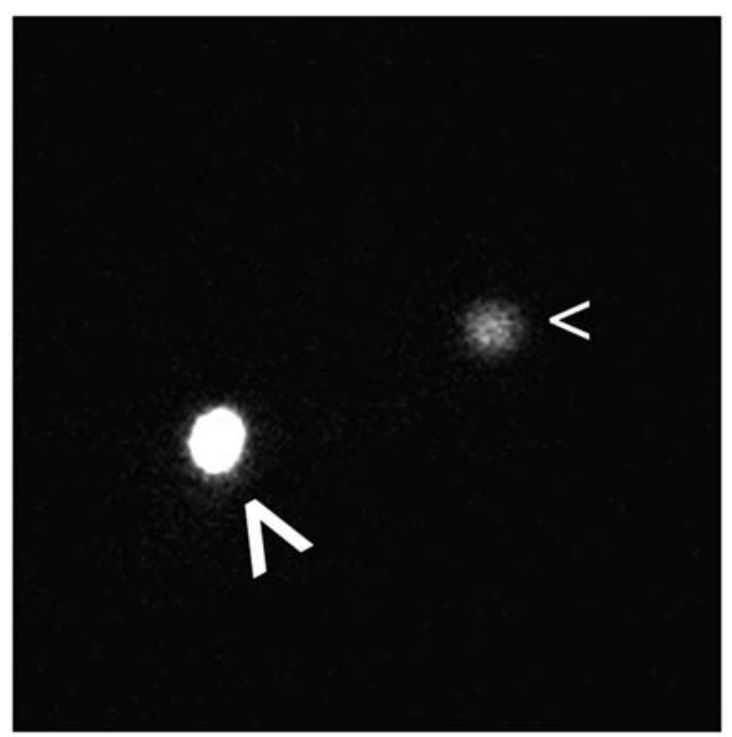

Fig. 2. Lymphoscintigraphy of the left breast: the large arrow indicates the injection site of the radiotracer in the periareolar region of the upper outer quadrant. After about $30 \mathrm{~min}$, the SLN can be identified at the anterior pilaster of the axillary cavity (small arrow). 
36 of the 38 patients, preoperative lymphoscintigraphy did not identify the SLNs, which were, however, identified intraoperatively.

In four cases of failure to find the SLN, axillary lymphadenectomy was performed in any case on principle.

The average number of detected SLNs was $1.88 \pm 0.9$ in group A and $1.91 \pm 0.7$ in group B. The number of metastatic SLNs was $69(20.3 \%)$ in group A and $114(19.8 \%)$ in group B. The average number of detected SLNs and the incidence of positive SLNs were not significantly different between the groups of patients (chi-square $0.29 ; P=0.59$ ).

\section{Discussion}

Various sites of injection including the PT, subdermal, intradermal, and PA have been described, but the optimal site of tracer injection has long been a matter of debate.

The first technique used in SLB was PT and this has become common in a clinical setting. Nevertheless, there are some difficulties involved by this method, particularly regarding the operator's experience in making the correct radiotracer injection, and also in the case of non-palpable tumors, where ultrasound or stereotactic guidance is required.

There are also other problems related to the fact that it is necessary to perform several injections in the peritumoral site, since the lymphatic structures, depending on their depth, may be represented in varying degrees, and may also be infiltrated by the tumor with reduced progression of the tracer, which will tend to accumulate at the injection site. For these reasons this approach has been criticized especially in non-palpable and multicentric tumors and in those lesions located in the upper outer quadrant because of the shine-through phenomenon determining high background noise in the axilla.

Subdermal PA injection of the radiotracer, more recently introduced, is a simple and easy method that does not require extensive experience (19). Several studies have emphasized the importance of the subareolar lymphatic plexus on the basis of the high number of lymphatic tracts in this area compared with those in the breast parenchyma.

This finding may be explained by the embryologic development of the breast from the epithelium of the primitive milk streak. During development, lymphatic vessels elongate and grow synchronously in parallel with the growth of the lactiferous ducts, but they stay connected with the subareolar plexus. The subareolar plexus communicates with superficial and deep intramammary channels and drains into the regional lymph nodes via one or two large lymph trunks. These channels maintain their connection to the SA lymphatic plexus in the mature breast.

The hypothesis of the existence of a common plexus between deep and periareolar lymphatic vessels has been confirmed by several other authors $(20,21)$.

In our retrospective study, we compared the lymphatic flow in the breast using the two injection sites PT and subdermal PA in two series of patients. In accordance with other authors, the results obtained confirm that the entire breast, including the nipple-areola complex, drains into the same axillary lymph nodes (22-24). With subdermal PA injection a higher number of SLNs is identified $(1.88 \pm 0.9$ vs. $1.91 \pm 0.7)$ but it is most important to identify the node that is the first draining step of the tumor tissue, and lymphoscintigraphy is able to identify it with accuracy. In our experience, the rate of positive nodes is not significantly different in the two groups of patients.

Several authors state that PA injections detect fewer extra-axillary nodes than the PT injection $(10,11)$. Borgstein $e t$ al. have also admitted that the only possible disadvantage of the PA injection lies in its inability to permit detection of accessory lymphatic pathways, especially with regard to the internal mammary chain (21).

Our experience confirms this and, in fact, with the PA injection we did not find any nodes outside the axillary site, compared with the PT injection, where we found 4/796 internal mammary chain nodes $(0.5 \%)$. Nevertheless, it must also be born in mind that most surgeons do not remove extra-axillary nodes routinely. However, the treatment of internal mammary chain nodes is a matter of debate; some authors affirm that dissection of the internal mammary chain lymph nodes benefits only a small portion of breast cancer patients, and would result in more injures to these patients and lead to more complications (25-27). Several other authors are of a completely different opinion, stating that the therapy was altered in a substantial proportion of patients by internal mammary SLNB and survival was worse in patients who had internal mammary drainage that was not taken into account when adjuvant therapy was planned $(28,29)$.

In our opinion, there are not enough published works on the subject, and more large clinical trials are necessary in order to explore the value of internal mammary node biopsy.

Finally, a slight improvement in the sensitivity of lymphoscintigraphy occurred in Group B patients compared with the Group A (90.85 vs. 93.39); in our opinion, this was due to the optimization of the technique by the medical staff concerned.

The results obtained confirm the hypothesis that the lymphatic pathways draining different areas of the 
breast parenchyma, and the subareolar plexus leads to the same sentinel lymph node. PT versus PA injection of radiotracer showed comparable success rates for axillary SLNs identification. PA injection of radiotracer showed comparable success rates for axillary SLNs identification.

In conclusion, we believe that the PA injection is to be considered the method of choice in the identification of SLNs. In fact, it can be considered a rapid and reliable method, with a low radiation dose for the patients that does not require either extensive experience on the part of the operator and or the use of ultrasound or stereotactic guidance in peritumoral injection for identifying SLNs in breast cancer patients. It is easy to perform, especially in non-palpable breast cancer.

\section{Funding}

This research received no specific grant from any funding agency in the public, commercial, or not-for-profit sectors.

\section{References}

1. Giuliano AE, Kirgan DM, Guenther JM, et al. Lymphatic mapping and sentinel lymphadenectomy for breast cancer. Ann Surg 1994;220:391-398.

2. Morton DL, Wen DR, Wong JH, et al. Technical details of intraoperative lymphatic mapping for early stage melanoma. Arch Surg 1992;127:392-399.

3. Van der Veen H, Hoekstra OS, Paul MA, et al. Gamma probe-guided sentinel node biopsy to select patients with melanoma for lymphadenectomy. $\mathrm{Br} \quad \mathrm{J}$ Surg 1994;81:1769-1770.

4. Ciafrocca M, Goldstein LJ. Prognostic and predictive factors on early-stage breast cancer. Oncologist 2004;9:606.

5. Banerjee M, George J, Song EY, et al. Tree-based model for breast cancer prognostication. $J$ Clin Oncol 2004;22:2567-2575.

6. Veronesi U, Paganelli G, Viale G, et al. Sentinel lymph node biopsy and axillary dissection in breast cancer: results in a large series. $\mathbf{J}$ Natl Cancer Inst 1999;91:368-373.

7. Posther KE, Wilke LG, Giuliano AE. Sentinel lymph node dissection and the current status of American trials on breast lymphatic mapping. Semin Oncol 2004;31:426-436.

8. Wang Y, Yu J, Wang L. The study of sentinel node biopsy in clinical node negative breast cancer in China. Breast Cancer Res Treat 2004;88:S89.

9. McMasters KM, Giuliano AE, Ross MI, et al. Sentinellymph-node biopsy for breast cancer-not yet the standard of care. N Engl J Med 1998;339:990-995.

10. van der Ploeg I, Tanis PJ, Valdes Olmos RA, et al. Breast cancer patients with extra-axillary sentinel nodes only may be spared axillary lymph node dissection. Ann Surg Oncol 2008;15:3239-3243.
11. Estourgie SH, Nieweg OE, Olmos RA, et al. Lymphatic drainage patterns from the breast. Ann Surg 2004;239:232-237.

12. Anagnostou C, Baiocco C, Arnulfo A, et al. The value of imaging in standing position in preoperative breast lymphoscintigraphy. Clin Nucl Med 2011;36:683-688.

13. Leidenius MH. Sentinel node biopsy in breast cancer. Acta Radiol 2005;46:791-801.

14. Rodier JF, Velten M, Wilt M, et al. Prospective multicentric randomized study comparing periareolar and peritumoral injection of radiotracer and blue dye for the detection of sentinel lymph node in breast sparing procedures: FRANSENODE trial. $\mathrm{J}$ Clin Oncol 2007;20:3664-3669.

15. Shimazu K, Tamaki Y, Uchi T, et al. Comparison between periareolar and peritumoral injection of radiotracer for sentinel lymph node biopsy in patients with breast cancer. Surgery 2002;131:277-286.

16. Sandrucci S, Sorba Casalegno $\mathrm{P}$, Percivale $\mathrm{P}$, et al. Sentinel lymph node mapping and biopsy for breast cancer: a review of the literature relative to 4791 procedures. Tumori 1999;85:425-434.

17. Kern KA. Sentinel lymph node mapping in breast cancer using subareolar injection of blue dye. J Am Coll Surg 1999;189:539-545.

18. Smith LF, Cross MJ, Klimberg VS. Subareolar injection is a better technique for sentinel lymph node biopsy. Am J Surg 2000;180:434-438.

19. Leikola JP, Leppänen EA, von Smitten KA, et al. A second radioisotope injection enhances intraoperative sentinel node identification in breast cancer patients without visualized nodes on preoperative lymphoscintigraphy. Acta Radiol 2006;47:760-763.

20. Tanis PJ, Nieweg OE, Olmos RA, et al. Anatomy and physiology of lymphatic drainage of the breast from the perspective of sentinel node biopsy. J Am Coll Surg 2001;192:399-404.

21. Borgstein PJ, Meijer S, Pijpers RJ, et al. Functional lymphatic anatomy for sentinel node biopsy in breast cancer: echoes from the past and the periareolar blue method. Ann. Surg 2000;232:81-89.

22. Kargozaran H, Shah M, Li Y, et al. Concordance of peritumoral technetium $99 \mathrm{~m}$ colloid and subareolar blue dye injection in breast cancer sentinel lymph node biopsy. J Surg Res 2007;143:126-129.

23. Garcia-Manero M, Olartecoechea B, Royo P. Different injection sites of radionuclide for sentinel lymph node detection in breast cancer: single institution experience. Eur J Obstet Gynecol Reprod Biol 2010;153:185-187.

24. Bauer TW, Spitz FR, Callans LS, et al. Subareolar and peritumoral injection identify similar sentinel nodes for breast cancer. Ann Surg Oncol 2002;9:169-176.

25. Klauber-DeMore N, Bevilacqua JL, Van Zee KJ, et al. Comprehensive review of the management of internal mammary lymph node metastases in breast cancer. J Am Coll Surg 2001;193:547-555.

26. Chen RC, Lin NU, Golshan M, et al. Internal mammary nodes in breast cancer: diagnosis and implications for 
patient management - a systematic review. J Clin Oncol 2008;26:4981-4989.

27. Domenech-Vilardell A, Bajen MT, Benitez AM, et al. Removal of the internal mammary sentinel node in breast cancer. Nucl Med Commun 2009;30:962-970.

28. Paredes P, Vidal-Sicart S, Zanon G, et al. Clinical relevance of sentinel lymph nodes in the internal mammary chain in breast cancer patients. Eur J Nucl Med Mol Imaging 2005;32:1283-1287.

29. Madsen E, Gobardhan P, Bongers V, et al. The impact on post surgical treatment of sentinel lymph node biopsy of internal mammary lymph nodes in patients with breast cancer. Ann Surg Oncol 2007;14:1486-1492. 
Copyright of Acta Radiologica is the property of Sage Publications, Ltd. and its content may not be copied or emailed to multiple sites or posted to a listserv without the copyright holder's express written permission. However, users may print, download, or email articles for individual use. 